\title{
Practical stability with respect to a part of variables of stochastic differential equations
}

\author{
Tomás Caraballo $^{a *}$ Faten Ezzine $^{b}$

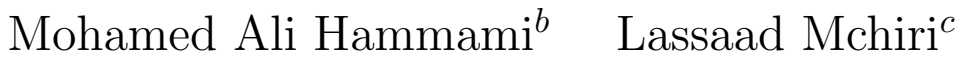 \\ ${ }^{a}$ Universidad de Sevilla, \\ Depto. Ecuaciones Diferenciales y Análisis Numérico, \\ Facultad de Matemáticas, Sevilla (Spain) \\ ${ }^{b}$ University of Sfax, Faculty of Sciences of Sfax, Department of Mathematics, Tunisia \\ \{E.mail: MohamedAli.Hammami@fss.rnu.tn\}
}

\begin{abstract}
In this paper, practical stability with respect to a part of the variables of nonlinear stochastic differential equations (SDEs) are studied.

We investigate, the global practical uniform asymptotic, the global practical uniform pth moment exponential stability, as well as the global practical uniform exponential stability with respect to a part of the variables based on Lyapunov techniques. We provide also some illustrative examples to show the usefulness of the notion of stability with respect to a part of variables for SDEs.
\end{abstract}

Keywords: Stochastic systems, Lyapunov techniques, Itô formula, Brownian motion, nontrivial solution, practical stability with respect to a part of the variables .

\section{Introduction}

Stability of stochastic differential equations (SDEs) has become a very prevalent theme of recent research in mathematics and its applications. Stochastic model can be used to solve the problem

\footnotetext{
*The research of Tomás Caraballo has been partially supported by FEDER and Ministerio de Economía y Competitividad (Spain) under grant MTM2011-22411
} 
which evinces by accident, noise, etc.

This paper aims mainly to establish some criteria for the global practical uniform asymptotic, the global practical uniform pth moment exponential stability, and the global practical uniform exponential stability with respect to a part of the variables of a class of nonlinear stochastic differential equations (SDEs) of the form:

$$
d x(t)=f(x(t), t) d t+g(x(t), t) d B(t) .
$$

In this way, considerable attention has been paid to the concept of stability with respect to a part of the system's states. Such concept arises from the study of electro-magnetics [23], inertial navigation systems [22], spacecraft stabilization via gimballed gyroscopes and/or flywheels [24], combustion systems [3], vibrations in rotating machinery [20], and biocenology [21].

The method of Lyapunov functions is one of the most powerful tool to study the stability of stochastic dynamical system, with the emergence of the second method of Lyapunov Peiffer and Rouche (1969), Rouche et al. (1977), Rumyantsev (1957), Rumyantsev and Oziraner (1987), Savchenko and Ignatyev (1989), Sontag and Wang (2001), Vorotnikov (1998), Vorotnikov and Rumyantsev (2001), involved the notion of stability with respect to a part of the variables especially when the origin considered as an equilibrium point. We would like to mention here the references [7],[12],[19],[24], among others.

Stability with respect to a part of the variables, has been used in investigating the qualitative properties of equilibria and boundedness properties of motions of dynamical systems determined by ordinary differential equations, difference equations, functional differential equations, stochastic differential equations, etc. It involves a notion of stability with respect to only a prespecified subset of the state variables characterizing the motions of the system under investigation.

Several interesting and important variants to Lyapunov's original concepts of practical stability were proposed in [1]-[4]-[10]. When the origin is not necessarily an equilibrium point, we can study the stability with respect to a part of the variables of the SDE in a small neighborhood of the origin in terms of convergence of solution in probability with respect to a part of the variables to a small ball.

Our main objective in this paper is to highlight situations in which the results in [10] cannot be applied to obtain the global practical stability in all variables but at least we can prove for some of the variables.

The remainder of this paper is organized as follows. In section 2, we give necessary preliminaries and results. In sections 3,4 , and 5 , we give sufficient conditions to ensure the global practical uniform asymptotic, the global practical uniform pth moment exponential stability, and the global practical uniform exponential stability with respect to a part of the variables of SDEs. Moreover, we exhibit some illustrative examples to show the applicability of the main results. 


\section{Auxiliary Facts and Results}

Consider the following $n$-dimensional stochastic differential equation (SDE):

$$
d x(t)=f(x(t), t) d t+g(x(t), t) d B_{t}, \quad \forall t \geq 0 .
$$

Where $f: \mathbb{R}^{n} \times \mathbb{R}_{+} \longrightarrow \mathbb{R}^{n}, g: \mathbb{R}^{n} \times \mathbb{R}_{+} \longrightarrow \mathbb{R}^{n \times m}, x=\left(x_{1}, \ldots ., x_{n}\right)^{T}$ and $B_{t}=\left(B_{1}(t), \ldots ., B_{m}(t)\right)^{T}$ is an $m$-dimensional Brownian motion defined on a complete probability space $(\Omega, \mathcal{F}, \mathbb{P})$.

We assume that there exists $t$ such that $f(0, t) \neq 0$ or $g(0, t) \neq 0$, i.e. the stochastic differential equation (2.1) does not have the trivial solution $x=0$.

The functions $f(x(t), t)$ and $g(x(t), t)$ verify the following standard assumptions for Itô calculus [16].

$$
\begin{array}{ll}
\int_{0}^{T}\|f(x(s), s)\| d s<\infty \quad \text { a.s. } & \forall T>0, \\
\int_{0}^{T}\|g(x(s), s)\|^{2} d s<\infty \quad \text { a.s. } \quad \forall T>0 .
\end{array}
$$

We assume that both $f$ and $g$ satisfy the following conditions:

$$
\begin{gathered}
\|f(x, t)\|^{2}+\|g(x, t)\|^{2} \leq K_{1}\left(1+\|x\|^{2}\right), \text { for all } t \geq 0, x \in \mathbb{R}^{n}, \\
\|f(x, t)-f(y, t)\| \vee\|g(x, t)-g(y, t)\| \leq K_{2}\|x-y\|, \text { for all } t \geq 0, x, y \in \mathbb{R}^{n},
\end{gathered}
$$

where $k_{1}$ and $k_{2}$ are given positive reals.

Denote $x=(y, z)^{T}$ where $y=\left(y_{1}, \ldots ., y_{n_{1}}\right)^{T} \in \mathbb{R}^{n_{1}}, z=\left(z_{1}, \ldots, z_{n_{2}}\right)^{T} \in \mathbb{R}^{n_{2}}, n_{1}+n_{2}=n$;

$$
\|y\|=\sqrt{y_{1}^{2}+\ldots+y_{n_{1}}^{2}},\|z\|=\sqrt{z_{1}^{2}+\ldots+z_{n_{2}}^{2}},\|x\|=\left(\|y\|^{2}+\|z\|^{2}\right)^{\frac{1}{2}} .
$$

The SDE (2.1) has a unique global solution $x\left(t, t_{0}, x_{0}\right)$ with initial condition $x_{0}$ (Mao, 1997, Oksendal, 2003). In what follows we use $x\left(, t_{0}, x_{0}\right)$, or simply $x(t)$ for a solution on some small interval.

\section{Global practical uniform asymptotic stability of stochas- tic differential equations with respect to a part of the variables}

In this section, we study the asymptotic stability in probability with respect to a part of the variables of stochastic differential systems when the origin is not an equilibrium point.

Therefore, we will study the asymptotic stability with respect to $x_{1}$ of the SDE in a small neighborhood of the origin in terms of convergence of solution in probability with respect to a part of the variables to a small ball $B_{r}:=\left\{x \in \mathbb{R}^{n}:\|x\| \leq r\right\}, r>0$. 
Definition 3.1. The solution of (2.1) is said to be globally uniformly bounded in probability, if for each $\alpha>0$, there exists $c=c(\alpha)>0$ (independent of $t_{0}$ ) such that,

$$
\text { for every } t_{0} \geq 0 \text {, and all } x_{0} \in \mathbb{R}^{n} \text { with } \quad\left\|x_{0}\right\| \leq \alpha, \quad \sup _{t \geq t_{0}}\left\|x\left(t, t_{0}, x_{0}\right)\right\| \leq c(\alpha), \quad \text { a.s. }
$$

Definition 3.2. $(i)$ The ball $B_{r}$ is said to be uniformly stable with respect to $y$ in probability, if for each $\varepsilon \in] 0,1[$ and $k>r$, there exists $\delta=\delta(\varepsilon, k)>0$ such that

$$
\mathbb{P}\left(\left\|y\left(t, t_{0}, x_{0}\right)\right\|<k, \forall t \geq t_{0} \geq 0\right) \geq 1-\varepsilon \text { for all }\left\|x_{0}\right\|<\delta
$$

(ii) The ball $B_{r}$ is said to be globally uniformly stable with respect to $y$ in probability, if it is uniformly stable with respect to $y$ in probability and the solution of (2.1) is globally uniformly bounded in probability.

Definition 3.3. The ball $B_{r}$ is said to be globally uniformly attractive with respect to $y$ in probability, if for each $\varepsilon \in] 0,1\left[, k>r\right.$ and $c>0$ (independent of $t_{0}$ ), there exists $T=T(\varepsilon, c)>0$ such that, for all $t_{0} \geq 0$, it holds

$$
\mathbb{P}\left(\left\|y\left(t, t_{0}, x_{0}\right)\right\|<k, \forall t \geq t_{0}+T\right) \geq 1-\varepsilon \quad \text { for all } x_{0} \in \mathbb{R}^{n} \text { such that }\left\|x_{0}\right\|<c .
$$

Definition 3.4. The system (2.1) is said to be globally uniformly practically asymptotically stable with respect to $y$ in probability, if there exists $r>0$ such that $B_{r}$ is globally uniformly stable with respect to $y$ in probability and globally uniformly attractive with respect to $y$ in probability.

Definition 3.5 (Class $\mathcal{K}$ function). A continuous function $\alpha:[0, a) \rightarrow[0,+\infty)$ is said to belong to class $\mathcal{K}$, if it is strictly increasing and $\alpha(0)=0$. It is said to belong to class $\mathcal{K}_{\infty}$ if $a=+\infty$ and $\alpha(r) \rightarrow+\infty$ as $r \rightarrow+\infty$.

Definition 3.6. (see [24]) A function $V: \mathbb{R}_{+} \times \mathbb{R}^{n} \rightarrow \mathbb{R}$ is said to be positive definite with respect to $y$ (or y-positive definite) if there exist $\alpha_{1} \in \mathcal{K}$ such that $V(t, x) \geq \alpha_{1}(\|y\|)$.

Definition 3.7. (see [24]) A function $V: \mathbb{R}_{+} \times \mathbb{R}^{n} \rightarrow \mathbb{R}$ is said to have an infinitesimal upper limit in $\mathrm{y}$ if there exists $\alpha_{2} \in \mathcal{K}$ such that $V(t, x) \leq \alpha_{2}(\|y\|)$.

We will define now $C^{2,1}\left(\mathbb{R}^{n} \times \mathbb{R}_{+}, \mathbb{R}\right)$ the family of all real-valued functions $V(x, t)$ defined on $\mathbb{R}^{n} \times \mathbb{R}_{+}$which are twice continuously differentiable in $x$ and once in $t$.

If $V \in C^{2,1}\left(\mathbb{R}^{n} \times \mathbb{R}_{+}, \mathbb{R}\right)$, we set

$V_{t}(x, t)=\frac{\partial V}{\partial t}(x, t) ; V_{x}(x, t)=\left(\frac{\partial V}{\partial y}(x, t), \frac{\partial V}{\partial z}(x, t)\right) ; V_{x x}(x, t)=\left(\frac{\partial^{2} V}{\partial x_{i} \partial x_{j}}(x, t)\right)_{n \times n}$.

Define the differential operator $\mathcal{L}$ as associated with equation (2.1) by

$$
L V(x, t)=V_{t}(x, t)+V_{x}(x, t) f(x, t)+\frac{1}{2} \operatorname{trace}\left[g(x, t)^{T} V_{x x}(x, t) g(x, t)\right] .
$$


By Itô's formula, it follows

$$
d V(x(t), t)=L V(x(t), t) d t+V_{x}(x(t), t) g(x(t), t) d B_{t} .
$$

Our first main result in this section reads as follow.

Theorem 3.1. Assume that there exist $V \in C^{2,1}\left(\mathbb{R}^{n} \times \mathbb{R}_{+}, \mathbb{R}_{+}\right), \alpha_{1}, \alpha_{2} \in \mathcal{K}_{\infty}$, with $\alpha_{1}: \mathbb{R}_{+}^{*} \rightarrow \mathbb{R}_{+}^{*}$ and $\alpha_{3} \in \mathcal{K}, M>0$, such that for all $t \geq 0$, and all $x \in \mathbb{R}^{n}$,

$$
\begin{gathered}
\alpha_{1}(\|y\|) \leq V(x, t) \leq \alpha_{2}(\|y\|), \\
L V(x, t) \leq-\alpha_{3}(\|y\|)+\varphi(t),
\end{gathered}
$$

where $\varphi(t)$ is a continuous nonnegative function with

$$
\lim _{t \rightarrow+\infty} \varphi(t)=0
$$

and

$$
\int_{0}^{+\infty} \varphi(t) d t \leq M<+\infty
$$

Furthermore, we suppose that for all $t \geq t_{0} \geq 0,\left\|z\left(t, t_{0}, x_{0}\right)\right\| \leq h, \quad$ a.s. where $h$ is a positive constant independent of $x_{0}$ and $t_{0}$.

Then, system (2.1) is globally uniformly practically asymptotically stable with respect to $y$ in probability.

Remark 3.2. If $\varphi(t)$ is uniformly continuous nonnegative function, then By Barbalat's Lemma (Khalil, 2002) (3.7) implies (3.6).

Proof. Let us start prove the uniform stability of $B_{r}$ with respect to $y$ in probability.

Let $\varepsilon \in] 0,1[$, and take a small positive real number $r$, and $k>r$.

Assume that there exists $\delta=\delta(\varepsilon, k) \in] 0, k\left[\right.$ such that, for $x_{0} \in S_{\delta}, S_{\delta}=\left\{x \in \mathbb{R}^{n} /\|x\|<\delta\right\}$ and $\alpha_{1}(k)>\frac{M}{\varepsilon}$, we deduce

$$
\frac{V\left(x_{0}, 0\right)}{\varepsilon} \leq \alpha_{1}(k)-\frac{M}{\varepsilon} .
$$

Define the stopping time

$$
\tau_{k}=\inf \left\{t \geq 0 ;\left\|y\left(t ; t_{0}, x_{0}\right)\right\| \geq k\right\} .
$$

By Itô's formula, for any $t \geq 0$,

$$
V\left(x\left(\tau_{k} \wedge t\right), \tau_{k} \wedge t\right)=V\left(x_{0}, 0\right)+\int_{0}^{\tau_{k} \wedge t} L V(x(s), s) d s+\int_{0}^{\tau_{k} \wedge t} V_{x}(x(s), s) g(x(s), s) d B_{s} .
$$


Taking the expectation on both sides, we obtain that $0 \leq \mathbb{E}\left(V\left(x\left(\tau_{k} \wedge t\right), \tau_{k} \wedge t\right)\right)=V\left(x_{0}, 0\right)+\mathbb{E}\left(\int_{0}^{\tau_{k} \wedge t} L V(x(s), s)\right) d s$.

This, together with (3.5) and (3.7), implies

$$
\begin{aligned}
\mathbb{E}\left(V\left(x\left(\tau_{k} \wedge t\right), \tau_{k} \wedge t\right)\right) & \leq V\left(x_{0}, 0\right)+\mathbb{E}\left(\int_{0}^{\tau_{k} \wedge t} \varphi(s)-\alpha_{3}(\|y(s)\|) d s\right) \\
& \leq V\left(x_{0}, 0\right)+\mathbb{E}\left(\int_{0}^{\tau_{k} \wedge t} \varphi(s) d s\right) \\
& \leq V\left(x_{0}, 0\right)+\mathbb{E}\left(\int_{0}^{+\infty} \varphi(s) d s\right) \\
& \leq V\left(x_{0}, 0\right)+M \\
& \leq \varepsilon \alpha_{1}(k) .
\end{aligned}
$$

Note that, $\left\|y\left(\tau_{k} \wedge t ; t_{0}, x_{0}\right)\right\|=\left\|y\left(\tau_{k} ; t_{0}, x_{0}\right)\right\|=k$ if $\tau_{k} \leq t$.

Hence,

$$
\begin{aligned}
\mathbb{E}\left(V\left(x\left(\tau_{k} \wedge t\right), \tau_{k} \wedge t\right)\right) & \geq \mathbb{E}\left(1_{\left\{\tau_{k} \leq t\right\}} V\left(x\left(\tau_{k}\right), \tau_{k}\right)\right) \\
& \geq \mathbb{E}\left(1_{\left\{\tau_{k} \leq t\right\}} \alpha_{1}\left(\left\|y\left(\tau_{k} ; t_{0}, x_{0}\right)\right\|\right)\right) \\
& \geq \mathbb{E}\left(1_{\left\{\tau_{k} \leq t\right\}} \alpha_{1}(k)\right) \\
& =\alpha_{1}(k) \mathbb{E}\left(1_{\left\{\tau_{k} \leq t\right\}}\right) \\
& =\alpha_{1}(k) \mathbb{P}\left(\tau_{k} \leq t\right) .
\end{aligned}
$$

Consequently,

$$
\alpha_{1}(k) \mathbb{P}\left(\tau_{k} \leq t\right) \leq \varepsilon \alpha_{1}(k)
$$

This implies immediately that,

$$
\mathbb{P}\left(\tau_{k} \leq t\right) \leq \varepsilon
$$

Letting, $t \rightarrow+\infty$, we get $\mathbb{P}\left(\tau_{k}<+\infty\right) \leq \varepsilon$.

That is, for all $\varepsilon \in] 0,1[, r \geq 0$ and $k>r$, there exists $\delta=\delta(\varepsilon, k) \in] 0, k[$, such that

$$
\mathbb{P}\left(\left\|y\left(t ; t_{0}, x_{0}\right)\right\|<k, \forall t \geq t_{0} \geq 0\right) \geq 1-\varepsilon, \quad \text { for all }\left\|x_{0}\right\|<\delta .
$$

Let us now prove the global uniform attractivity of $B_{r}$ with respect to $y$ in probability.

It is sufficient to prove that, for each $\varepsilon \in] 0,1[, \lambda>r$ and $c>0$, there exists $T=T(\varepsilon, c)>0$ (independent of $t_{0}$ ), such that for all $t \geq t_{0}+T$, we have

$$
\mathbb{P}\left(\left\|y\left(t ; t_{0}, x_{0}\right)\right\|<\lambda\right) \geq 1-\varepsilon, \text { for all }\left\|x_{0}\right\|<c .
$$

Let $\varepsilon \in] 0,1\left[, h>r\right.$ large enough and $c>0$ (independent of $t_{0}$ ), such that $\left\|x_{0}\right\|<c$ and $\alpha_{1}(\lambda)>\frac{M}{\varepsilon}$, we deduce

$$
\alpha_{1}(h)-\frac{M}{\varepsilon} \geq \frac{V\left(x_{0}, 0\right)}{\varepsilon} .
$$


Suppose now that there exists $T=T(\varepsilon, c)>0$ such that, for all $t_{0} \geq 0$,

$$
\tau_{\lambda}=\inf \left\{t \geq t_{0}+T ;\left\|y\left(t ; t_{0}, x_{0}\right)\right\| \geq \lambda\right\}
$$

By the Itô formula, for any $t \geq t_{0}+T$, we obtain

$$
0 \leq \mathbb{E}\left(V\left(x\left(\tau_{\lambda} \wedge t\right), \tau_{\lambda} \wedge t\right)\right)=V\left(x_{0}, 0\right)+\mathbb{E}\left(\int_{0}^{\tau_{\lambda} \wedge t} L V(x(s), s)\right) d s .
$$

Taking the expectation on both sides and making use (3.5) and (3.7), we obtain that

$$
\begin{aligned}
\mathbb{E}\left(V\left(x\left(\tau_{\lambda} \wedge t\right), \tau_{\lambda} \wedge t\right)\right) & \leq V\left(x_{0}, 0\right)+\mathbb{E}\left(\int_{0}^{\tau_{\lambda} \wedge t}\left(\varphi(s)-\alpha_{3}(\|y(s)\|) d s\right)\right. \\
& \leq V\left(x_{0}, 0\right)+M \\
& \leq \varepsilon \alpha_{1}(h) .
\end{aligned}
$$

Note that, $\left\|y\left(\tau_{\lambda} \wedge t ; t_{0}, x_{0}\right)\right\|=\left\|y\left(\tau_{\lambda} ; t_{0}, x_{0}\right)\right\|=\lambda$ when $\tau_{\lambda} \leq t$. Therefore, we can obtain

$$
\begin{aligned}
\mathbb{E}\left(V\left(x\left(\tau_{\lambda} \wedge t\right), \tau_{\lambda} \wedge t\right)\right) & \geq \mathbb{E}\left(1_{\left\{\tau_{\lambda} \leq t\right\}} V\left(x\left(\tau_{\lambda}\right), \tau_{\lambda}\right)\right) \\
& \geq \mathbb{E}\left(1_{\left\{\tau_{\lambda} \leq t\right\}} \alpha_{1}\left(\left\|y\left(\tau_{\lambda} ; t_{0}, x_{0}\right)\right\|\right)\right) \\
& \geq \mathbb{E}\left(1_{\left\{\tau_{\lambda} \leq t\right\}} \alpha_{1}(\lambda)\right) \\
& =\alpha_{1}(\lambda) \mathbb{E}\left(1_{\left\{\tau_{\lambda} \leq t\right\}}\right) \\
& =\alpha_{1}(\lambda) \mathbb{P}\left(\tau_{\lambda} \leq t\right) .
\end{aligned}
$$

Then,

$$
\alpha_{1}(\lambda) \mathbb{P}\left(\tau_{\lambda} \leq t\right) \leq \varepsilon \alpha_{1}(\lambda)
$$

which implies that,

$$
\mathbb{P}\left(\tau_{\lambda} \leq t\right) \leq \varepsilon
$$

Letting $t \rightarrow \infty$ we get $\mathbb{P}\left(\tau_{\lambda}<+\infty\right) \leq \varepsilon$.

That is, for any $\varepsilon \in] 0,1\left[, \lambda>r\right.$ and $c>0$ (independent of $t_{0}$ ), for a fixed $T=T(\varepsilon, c)>0$, we have, for all $t \geq t_{0}+T, \mathbb{P}\left(\left\|y\left(t ; t_{0}, x_{0}\right)\right\|<\lambda ; \forall t \geq t_{0}+T\right) \geq 1-\varepsilon$, for all $\left\|x_{0}\right\|<c$.

For a small $r \in] 0, \delta\left[\right.$ and $\left\|x_{0}\right\|<r$, we can take $k=k(r)>0$, such that for all $t \geq t_{0} \geq 0$, we have $\left\|y\left(t ; t_{0}, x_{0}\right)\right\|<k$, a.s, for all $\left\|x_{0}\right\|<r$.

We also have for all $t \geq t_{0} \geq 0,\left\|z\left(t ; t_{0}, x_{0}\right)\right\|<h$, a.s.

Consequently, there exist $m=m(r)$ such that,

$$
\sup _{t \geq t_{0}}\|x(t)\|<m \quad \text { for all }\left\|x_{0}\right\|<r \quad \text { a.s. }
$$

Hence, the system (2.1)is globally uniformly practically asymptotically stable with respect to $y$ in probability.

We present an example that implement the previous Theorem. 
Example 3.3. Sorry Mohamed, in my opinion this example is not valid to justify that the results in our paper [10], namely Theorem 2.1, cannot be applied to all the variables and therefore the current result in this paper is meaningful. Let me explain: first, in the example below there are some mistakes, please check it carefully. Second, if we consider $V:=\frac{x_{1}^{2}+x_{2}^{2}+x_{3}^{2}}{2}$, then the result Theorem 2.1 in ref. [10] holds and therefore we have stability in the three variables. In my opinion, to show the applicability of the theory in the current paper, we need to find an example which is not stable in all the variables and that we can prove stability in some of the variables. If we cannot provide such example, then our results do not have any interest. Can you please think about this? This is the real motivation and importance of the present study in this paper.

Consider the following stochastic system

$$
\left\{\begin{array}{l}
d x_{1}(t)=\left(-x_{1}+\frac{1}{\sqrt{c h(t)}}\right) d t+\sqrt{2} x_{1} \sqrt{x_{2}^{4}+1} d B_{t} \\
d x_{2}(t)=\left(-x_{2}-x_{1}^{2} x_{2}\left(x_{2}^{4}+1\right)\right) d t \\
d x_{3}(t)=-\beta x_{3} d t
\end{array}\right.
$$

where $\beta>0, x(t)=\left(x_{1}(t), x_{2}(t), x_{3}(t)\right)^{T} \in \mathbb{R}^{3}$ and $B(t)$ is one-dimensional Brownian motion. It is clear that $x_{2}\left(t, t_{0}, x_{0}\right)$ is stochastically bounded, in fact, $x_{2}\left(t, t_{0}, x_{0}\right)=x_{20}(t, \omega) e^{-\beta\left(t-t_{0}\right)}$.

Denote, $\quad V:=\frac{x_{1}^{2}+x_{2}^{2}}{2}$.

Applying the Itô formula on $V$ leads to

$$
\begin{aligned}
L V & =x_{1}\left(-x_{1}+\frac{1}{\sqrt{c h(t)}}\right)-x_{2}^{2}-x_{1}^{2} x_{2}^{2}\left(x_{2}^{4}+1\right)+x_{1}^{2} x_{2}^{2}\left(x_{2}^{4}+1\right) \\
& =-x_{1}^{2}-x_{2}^{2}+x_{1} \frac{1}{\sqrt{c h(t)}} \\
& \leq-\frac{1}{2} x_{1}^{2}-x_{2}^{2}+\frac{1}{2} \frac{1}{\operatorname{ch}(t)} \\
& \leq-\frac{1}{2}\left(x_{1}^{2}+x_{2}^{2}\right)+\frac{1}{2} \frac{1}{\operatorname{ch}(t)}
\end{aligned}
$$

Note that we cannot apply theorem on practical asymptotic stability in probability [10], because all conditions of this Theorem are not fulfilled. But we can apply Theorem (3.1), since $V$ is positive definite with respect to $\left(x_{1}, x_{2}\right)$ and it have an infinitesimal upper limit in $\left(x_{1}, x_{2}\right)$. Furthermore,

$$
L V(x, t) \leq-\frac{1}{2}\left(x_{1}^{2}+x_{2}^{2}\right)+\varphi(t)
$$

where $\varphi(t)=\frac{1}{2} \frac{1}{c h(t)}$. which satisfied both conditions (3.6) and (3.7). 
Hence, all conditions of Thorem (3.1) are fulfilled and we get that (3.10) is globally uniformly practically asymptotically stable with respect to $\left(x_{1}, x_{2}\right)$ in probability.

\section{Global practical uniform pth moment exponential sta- bility of stochastic differential equation with respect to a part of the variables}

In this section, we present sufficient conditions for global practical pth moment exponential stability of SDE with respect to a part of the variables.

Definition 4.1. $i$ ) Let $p>0$, the ball $B_{r}$ is said to be globally uniformly pth moment exponentially stable with respect to $y$, if there exist $\lambda_{1}>0$ and $\lambda_{2}>0$, such that

$$
\mathbb{E}\left(\left\|y\left(t, t_{0}, x_{0}\right)\right\|^{p}\right) \leq \lambda_{1}\left\|x_{0}\right\|^{p} e^{-\lambda_{2}\left(t-t_{0}\right)}+r \quad \forall t \geq t_{0}, \quad \forall x_{0} \in \mathbb{R}^{n} .
$$

ii) The system (2.1) is said to be globally practically uniformly pth moment exponentially stable with respect to $y$ if there exists $r>0$ such that $B_{r}$ is globally uniformly pth moment exponentially stable with respect to $y$.

Within the method of Lyapunov, we present the following result.

Theorem 4.1. Assume that there exist $V \in C^{2,1}\left(\mathbb{R}^{n} \times \mathbb{R}_{+}, \mathbb{R}_{+}^{*}\right)$ and positive constants $c_{i}(i=$ $1,2,3), \gamma, p$, such that for all $x \neq 0$ and all $t \geq t_{0} \geq 0$,

$$
\begin{aligned}
& c_{1}\|y\|^{p} \leq V(x, t) \leq c_{2}\|y\|^{p}, \\
& L V(x, t) \leq-c_{3}\|y\|^{p}+\psi(t),
\end{aligned}
$$

where $\psi(t)$ is a continuous nonnegative function with

$$
\lim _{t \rightarrow+\infty} \psi(t)=0
$$

and

$$
\int_{t_{0}}^{+\infty} \psi(t) d t \leq \rho<+\infty .
$$

Furthermore, we suppose that for all $t \geq t_{0} \geq 0,\left\|z\left(t, t_{0}, x_{0}\right)\right\| \leq h, \quad$ a.s. where $h$ is a positive constant independent of $x_{0}$ and $t_{0}$.

Then, system (2.1) is globally practically uniformly pth moment exponentially stable with respect to $y$ with $r=\frac{\rho}{c_{1}}$.

In order to prove this Theorem, we need to recall an important Gronwall-Lemma see Dragomir [11] for other versions. 
Lemma 4.2. Let $b(t)$ and $f(t)$ be continuous functions for $t \geq t_{0} \geq 0$, let $v(t)$ be a differentiable function for $t \geq t_{0} \geq 0$, and suppose

$$
\dot{v}(t) \leq b(t) v(t)+f(t), \quad t \geq t_{0} \geq 0 .
$$

Then, for all $t \geq t_{0} \geq 0$,

$$
v(t) \leq v\left(t_{0}\right) \exp \left(\int_{t_{0}}^{t} b(s) d s\right)+\int_{t_{0}}^{t} f(s) \exp \left(\int_{s}^{t} b(\tau) d \tau\right) d s .
$$

Proof. For a fixed $t>t_{0}$ we have from (4.6) that

$$
\dot{v}(s) \leq b(s) v(s)+f(s), \quad s \in\left[t_{0}, t\right]
$$

Therefore

$$
\begin{aligned}
{[\dot{v}(s)-b(s) v(s)] \exp \left(\int_{s}^{t} b(\tau) d \tau\right) } & \leq f(s) \exp \left(\int_{s}^{t} b(\tau) d \tau\right), \quad s \geq t_{0}, \\
\frac{d}{d s}\left[v(s) \exp \left(\int_{s}^{t} b(\tau) d \tau\right)\right] & \leq f(s) \exp \left(\int_{s}^{t} b(\tau) d \tau\right) .
\end{aligned}
$$

By integration between $t_{0}$ and $t$

$$
v(t)-v\left(t_{0}\right) \exp \left(\int_{t_{0}}^{t} b(\tau) d \tau\right) \leq \int_{t_{0}}^{t} f(s) \exp \left(\int_{s}^{t} b(\tau) d \tau\right) d s
$$

and the result is shown.

Now we are able to prove our result Theorem (4.1).

Proof of Theorem 4.1. Applying the Itô formula on $V$ for any $t \geq t_{0} \geq 0$, leads to

$$
V(x(t), t)=V\left(x_{0}, t_{0}\right)+\int_{t_{0}}^{t} L V(x(s), s) d s+\int_{t_{0}}^{t} V_{x}(x(s), s) g(x(s), s) d B_{s} .
$$

Taking the expectation on both sides, we obtain that

$$
0 \leq \mathbb{E}(V(x(t), t))=V\left(x_{0}, t_{0}\right)+\int_{t_{0}}^{t} \mathbb{E}(L V(x(s), s)) d s .
$$

Differentiating this equality with respect to $t$ and taking into consideration conditions (4.2) and (4.3). We obtain

$$
\begin{aligned}
\frac{d}{d t} \mathbb{E}(V(x(t), t)) & =\mathbb{E}(L V(x(t), t)) \\
& \leq c_{3} \mathbb{E}\left(-\|y(t)\|^{p}\right)+\psi(t) \\
& \leq-\frac{c_{3}}{c_{2}} \mathbb{E}(V(t, x(t)))+\psi(t) .
\end{aligned}
$$


An application of lemma (4.2) this implies

$$
\begin{aligned}
\mathbb{E}(V(x(t), t)) & \leq V\left(x_{0}, t_{0}\right) e^{-\frac{c_{3}}{c_{2}}\left(t-t_{0}\right)}+\int_{t_{0}}^{t} \psi(s) e^{-\frac{c_{3}}{c_{2}}(t-s)} d s \\
& \leq c_{2}\left\|y_{0}\right\|^{p} e^{-\frac{c_{3}}{c_{2}}\left(t-t_{0}\right)}+\int_{t_{0}}^{+\infty} \psi(s) d s .
\end{aligned}
$$

Due to the fact that $\left\|y_{0}\right\| \leq\left\|x_{0}\right\|$ and condition (4.5), we deduce from the last inequality that

$$
\mathbb{E}(V(x(t), t)) \leq c_{2}\left\|x_{0}\right\|^{p} e^{-\frac{c_{3}}{c_{2}}\left(t-t_{0}\right)}+\rho .
$$

From (4.8), and $V(x(t), t) \geq c_{1}\|y\|^{p}$ for all $t \geq t_{0} \geq 0$, implies

$$
\begin{aligned}
\left.\mathbb{E}\left(\|y(t)\|^{p}\right)\right) & \leq \frac{1}{c_{1}} \mathbb{E}(V(x(t), t) \\
& \leq \frac{c_{2}}{c_{1}}\left\|x_{0}\right\|^{p} e^{-\frac{c_{3}}{c_{2}}\left(t-t_{0}\right)}+\frac{\rho}{c_{1}}
\end{aligned}
$$

Setting $\lambda_{1}=\frac{c_{2}}{c_{1}}$ and $\lambda_{2}=\frac{c_{3}}{c_{2}}$, we conclude that the system (2.1)is globally practically uniformly pth moment exponentially stable with respect to $y$ with $r=\frac{\rho}{c_{1}}$.

\section{Global practical uniform exponential stability of stochas- tic differential equation with respect to a part of the variables}

In this section, we are basically interested to study the global practical uniform exponential stability of SDE with respect to a part of the variables based on Lyapunov techniques.

Definition 5.1. $i$ ) The ball $B_{r}$ is said to be almost surely globally uniformly exponentially stable with respect to $y$ if for any $x_{0}$ such that $0<\left\|y\left(t, t_{0}, x_{0}\right)\right\|-r$, for all $t \geq 0$, it holds that

$$
\lim _{t \rightarrow \infty} \sup \frac{1}{t} \ln \left(\left\|y\left(t, t_{0}, x_{0}\right)\right\|-r\right)<0, \text { a.s. }
$$

ii) The system (2.1) is said to be almost surely globally practically uniformly exponentially stable with respect to $y$, if there exists $r>0$ such that $B_{r}$ is almost surely globally uniformly exponentially stable with respect to $y$.

Theorem 5.1. Assume that there exist a function $V \in C^{2,1}\left(\mathbb{R}^{d} \times \mathbb{R}_{+}, \mathbb{R}^{*}\right)$ and constants $p \in \mathbb{N}^{*}$, $\beta_{1} \geq 1, \gamma \geq \beta_{1}, \zeta \geq 0$ and $\beta_{2} \in \mathbb{R}, \beta_{3} \geq 0$ such that for all $t \geq t_{0} \geq 0$, and $x \in \mathbb{R}^{n}$, 
1. $\beta_{1}\|y\|^{p} \leq V(x, t)$

2. $L V(x, t) \leq \beta_{2} V(x, t)+\gamma$,

3. $\left\|V_{x}(x, t) g(x, t)\right\|^{2} \geq \varrho(t) V^{2}(x, t)+\zeta$,

where $\varrho(t)$ is a continuous non negative function with

$$
\lim _{t \longrightarrow+\infty} \sup \left(\frac{\int_{0}^{t} \varrho(s) d s}{t}\right) \leq \beta_{3} .
$$

Furthermore, we suppose that for all $t \geq t_{0} \geq 0, \quad\left\|z\left(t, t_{0}, x_{0}\right)\right\| \leq h, \quad$ a.s. where $h$ is a positive constant independent of $x_{0}$ and $t_{0}$.

Then,

$$
\lim _{t \rightarrow+\infty} \sup \frac{1}{t} \ln \left(\left\|y\left(t, t_{0}, x_{0}\right)\right\|-\left(\frac{\gamma}{\beta_{1}}\right)^{\frac{1}{p}}\right) \leq-\frac{\left[\left(\beta_{3}+\sigma\right)-2\left(\beta_{2}+1\right)\right]}{2}, \quad \text { a.s., for all } x_{0} \in \mathbb{R}^{n},
$$

where $\sigma$ is a positive constant.

In particular, if $\left(\beta_{3}+\sigma\right)>2\left(\beta_{2}+1\right)$, then the system (2.1) is said to be almost surely globally practically uniformly exponentially stable with respect to $y$, with $r=\left(\frac{\gamma}{\beta_{1}}\right)^{\frac{1}{p}}$.

In order to prove this Theorem we need to recall the following Lemma see Mao [16].

Lemma 5.2. For all $x_{0} \in \mathbb{R}^{n}$ such that $x_{0} \neq 0$ it holds

$$
\mathbb{P}\left(x\left(t ; t_{0}, x_{0}\right) \neq 0, \forall t \geq 0\right)=1 .
$$

That is, almost all the sample path of any solution starting from a non-zero state will never reach the origin.

Proof. Arguing by contradiction, if the lemma were false, there would exist some $x_{0} \neq 0$ such that $\mathbb{P}\{\tau<\infty\}>0$, where

$$
\tau=\inf \{t \geq 0 ; x(t)=0\}
$$

So, we can find a pair of constants $T>0$ and $\theta>1$, sufficiently large, such that $\mathbb{P}(B)>0$, where

$$
B=\{\tau \leq T \quad \text { and } \quad\|x(t)\| \leq \theta-1, \quad \text { for all } \quad 0 \leq t \leq \tau\} .
$$

But, by the standing hypotheses, there exist positive constants $K_{\theta}, r$, and a small positive constant $c$ such that

$$
\|f(x, t)\| \vee\|g(x, t)\| \leq K_{\theta}\|x\|+r \quad \text { for all } 0<c \leq\|x\| \leq \theta, \quad 0 \leq t \leq T
$$


Let $V(x, t)=\|x\|^{-1}$.

Then, by the Itô formula, for $0<c \leq\|x\| \leq \theta$ and $0 \leq t \leq T$,

$$
\begin{aligned}
L V(x, t) & =-\|x\|^{-3} x^{T} f(x, t)+\frac{1}{2}\left(-\|x\|^{-3}\|g(x, t)\|^{2}+3\|x\|^{-5}\left\|x^{T} g(x, t)\right\|^{2}\right) \\
& \leq\|x\|^{-2}\|f(x, t)\|+\|x\|^{-3}\|g(x, t)\|^{2} \\
& \leq\|x\|^{-2}\left(K_{\theta}\|x\|+r\right)+\|x\|^{-3}\left(K_{\theta}\|x\|+r\right)^{2} \\
& \leq K_{\theta}\left(1+K_{\theta}\right) V(x, t)+r\|x\|^{-1}\left(\|x\|^{-1}+2 K_{\theta}\|x\|^{-1}+r\|x\|^{-2}\right) \\
& \leq K_{\theta}\left(1+K_{\theta}\right) V(x, t)+r V(x, t)\left(\frac{1}{c}+\frac{2 K_{\theta}}{c}+\frac{r}{c^{2}}\right) \\
& \leq\left[K_{\theta}\left(1+K_{\theta}\right)+\beta\right] V(x, t),
\end{aligned}
$$

where, $\beta=r\left(\frac{1}{c}+\frac{2 K_{\theta}}{c}+\frac{r}{c^{2}}\right)$.

Now, for any $\varepsilon \in] 0,\left\|x_{0}\right\|[$, define the stopping time

$$
\tau_{\varepsilon}=\inf \{t \geq 0 ;\|x(t)\| \notin] \varepsilon, \theta[\} .
$$

Applying the Itô formula, we obtain

$$
\begin{aligned}
& \mathbb{E}\left[\exp \left\{-\left[K_{\theta}\left(1+K_{\theta}\right)+\beta\right]\left(\tau_{\varepsilon} \wedge T\right)\right\} V\left(x\left(\tau_{\varepsilon} \wedge T\right), \tau_{\varepsilon} \wedge T\right)\right]-V\left(x_{0}, 0\right), \\
& =\mathbb{E}\left[\int_{0}^{\tau_{\varepsilon} \wedge T} \exp \left\{-\left[K_{\theta}\left(1+K_{\theta}\right)+\beta\right] s\right\}\left[-\left(K_{\theta}\left(1+K_{\theta}\right)+\beta\right) V(x(s), s)+L V(x(s), s)\right] d s\right], \\
& \leq 0 .
\end{aligned}
$$

Note that for $\omega \in B$, this implies $\tau_{\varepsilon} \leq T$ and $\left\|x\left(\tau_{\varepsilon}\right)\right\|=\varepsilon$. The above inequality therefore involves that

$$
\mathbb{E}\left[\exp \left\{-\left[K_{\theta}\left(1+K_{\theta}\right)+\beta\right] T\right\} \varepsilon^{-1} 1_{B}\right] \leq\left\|x_{0}\right\|^{-1} .
$$

Hence,

$$
\mathbb{P}(B) \leq \varepsilon\left\|x_{0}\right\|^{-1} \exp \left\{\left[K_{\theta}\left(1+K_{\theta}\right)+\beta\right] T\right\} .
$$

Letting $\varepsilon \longrightarrow 0$ gives $\mathbb{P}(B)=0$, but this contradicts the definition of $B$. The proof is complete.

The next Lemma, whose proof can be found in [16], will be useful in our analysis:

Lemma 5.3. Let $g=\left(g_{1}, \ldots, g_{m}\right) \in L^{2}\left(\mathbb{R}_{+}, \mathbb{R}^{m}\right)$, and let $T, \alpha, \beta$ be any positive numbers. Then

$$
\mathbb{P}\left(\sup _{0 \leq t \leq T}\left[\int_{0}^{t} g(s) d B_{s}-\frac{\alpha}{2} \int_{0}^{t}\|g(s)\|^{2} d s\right]>\beta\right) \leq \exp (-\alpha \beta) .
$$


Now, we are able to prove our main result in this section.

\section{Proof of Theorem 5.1.}

Fix $x_{0} \neq 0$ in $\mathbb{R}^{n}$. By Lemma (5.2), $x(t) \neq 0$, for all $t \geq 0$ almost surely.

For any $y \in \mathbb{R}^{b}$, we obtain

$$
\begin{aligned}
\beta_{1}\|y\|^{p}-\gamma & =\beta_{1}\left(\|y\|^{p}-\frac{\gamma}{\beta_{1}}\right), \\
& =\beta_{1}\left(\|y\|^{p}-\left(\left(\frac{\gamma}{\beta_{1}}\right)^{\frac{1}{p}}\right)^{p}\right),
\end{aligned}
$$

and using

$$
a^{p}-b^{p}=(a-b)\left(a^{p-1}+a^{p-2} b+a^{p-3} b^{2}+\ldots+a^{0} b^{p-1}\right),
$$

equality (5.4) becomes

$$
\begin{aligned}
\beta_{1}\|y\|^{p}-\gamma & =\beta_{1}\left(\|y\|-\left(\frac{\gamma}{\beta_{1}}\right)^{\frac{1}{p}}\right)\left(\|y\|^{p-1}+\|y\|^{p-2}\left(\frac{\gamma}{\beta_{1}}\right)^{\frac{1}{p}}+\ldots+\left(\frac{\gamma}{\beta_{1}}\right)^{\frac{p-1}{p}}\right), \\
& \geq \beta_{1}\left(\|y\|-\left(\frac{\gamma}{\beta_{1}}\right)^{\frac{1}{p}}\right)\left(\frac{\gamma}{\beta_{1}}\right)^{\frac{p-1}{p}} .
\end{aligned}
$$

Since $\beta_{1} \geq 1$ and $\gamma \geq \beta_{1}$,we obtain

$$
V(x, t) \geq \beta_{1}\|y\|^{p} \geq \beta_{1}\|y\|^{p}-\gamma \geq \beta_{1}\left(\|y\|-\left(\frac{\gamma}{\beta_{1}}\right)^{\frac{1}{p}}\right)\left(\frac{\gamma}{\beta_{1}}\right)^{\frac{p-1}{p}} \geq\left(\|y\|-\left(\frac{\gamma}{\beta_{1}}\right)^{\frac{1}{p}}\right) .
$$

Thus,

$$
\left(\|y\|-\left(\frac{\gamma}{\beta_{1}}\right)^{\frac{1}{p}}\right) \leq V(x, t)
$$

and

$$
\ln \left(\|y\|-\left(\frac{\gamma}{\beta_{1}}\right)^{\frac{1}{p}}\right) \leq \ln (V(x, t)), \quad \forall t \geq 0, x \in \mathbb{R}^{n} .
$$

The Application of Itô formula, leads to, for all $t \geq 0$,

$$
d(\ln (V(x(t), t)))=\frac{L V(x(t), t)}{V(x(t), t)} d t+\frac{V_{x}(x(t), t) g(x(t), t)}{V(x(t), t)} d B_{t}-\frac{1}{2} \frac{\left\|V_{x}(x(t), t) g(x(t), t)\right\|^{2}}{V^{2}(x(t), t)} d t .
$$

That is,

$\int_{0}^{t} d(\ln (V(x(s), s))) d s=\int_{0}^{t} \frac{L V(x(s), s)}{V(x(s), s)} d s+\int_{0}^{t} \frac{V_{x}(x(s), s) g(x(s), s)}{V(x(s), s)} d B_{s}-\frac{1}{2} \int_{0}^{t} \frac{\left\|V_{x}(x(s), s) g(x(s), s)\right\|^{2}}{V^{2}(x(s), s)} d s$.

So,

$$
\ln (V(x(t), t))=\ln (V(x(0), 0))+\int_{0}^{t} \frac{L V(x(s), s)}{V(x(s), s)} d s+M(t)-\frac{1}{2} \int_{0}^{t} \frac{\left\|V_{x}(x(s), s) g(x(s), s)\right\|^{2}}{V^{2}(x(s), s)} d s .
$$


Where, $M(t)=\int_{0}^{t} \frac{V_{x}(x(s), s) g(x(s), s)}{V(x(s), s)} d B_{s}$, is a continuous martingale with initial value $M(0)=$ 0 .

Taking, into account the assumptions, we obtain that, for all $t \geq 0$

$$
\begin{aligned}
\ln (V(x(t), t)) & \leq \ln (V(x(0), 0))+\int_{0}^{t} \frac{\beta_{2} V(x(s), s)+\gamma}{V(x(s), s)} d s+M(t)-\frac{1}{2} \int_{0}^{t} \frac{\left\|V_{x}(x(s), s) g(x(s), s)\right\|^{2}}{V^{2}(x(s), s)} d s \\
& \leq \ln (V(x(0), 0))+\beta_{2} t+\int_{0}^{t} \frac{\gamma}{V(x(s), s)} d s+M(t)-\frac{1}{2} \int_{0}^{t} \frac{\left\|V_{x}(x(s), s) g(x(s), s)\right\|^{2}}{V^{2}(x(s), s)} d s \\
& \leq \ln (V(x(0), 0))+\beta_{2} t+\int_{0}^{t} \frac{\gamma}{\beta_{1}\|y(s)\|^{p}} d s+M(t)-\frac{1}{2} \int_{0}^{t} \frac{\left\|V_{x}(x(s), s) g(x(s), s)\right\|^{2}}{V^{2}(x(s), s)} d s \\
& \leq \ln (V(x(0), 0))+\beta_{2} t+t+M(t)-\frac{1}{2} \int_{0}^{t} \frac{\left\|V_{x}(x(s), s) g(x(s), s)\right\|^{2}}{V^{2}(x(s), s)} d s .
\end{aligned}
$$

That is,

$$
\ln (V(x(t), t)) \leq \ln (V(x(0), 0))+\left(\beta_{2}+1\right) t+M(t)-\frac{1}{2} \int_{0}^{t} \frac{\left\|V_{x}(x(s), s) g(x(s), s)\right\|^{2}}{V^{2}(x(s), s)} d s .
$$

Assign $\varepsilon \in] 0,1[$ arbitrarily and let $n=1,2, \ldots$ It follows from Lemma (5.3) that,

$$
\mathbb{P}\left\{\sup _{0 \leq t \leq n}\left[M(t)-\frac{\varepsilon}{2} \int_{0}^{t} \frac{\left\|V_{x}(x(s), s) g(x(s), s)\right\|^{2}}{V^{2}(x(s), s)} d s\right]>\frac{2}{\varepsilon} \ln (n)\right\} \leq \frac{1}{n^{2}} .
$$

Applying the Borel-Cantelli lemma we see that, for almost all $\omega \in \Omega$, there exists an integer $n_{0}=n_{0}(\omega)$, such that if $n \geq n_{0}$,

$$
M(t) \leq \frac{2}{\varepsilon} \ln (n)+\frac{\varepsilon}{2} \int_{0}^{t} \frac{\left\|V_{x}(x(s), s) g(x(s), s)\right\|^{2}}{V^{2}(x(s), s)} d s, \text { for all } 0 \leq t \leq n .
$$

Then, the inequality (5.5) becomes, for all $0 \leq t \leq n, n \geq n_{0}$ almost surely, as

$$
\begin{aligned}
\ln (V(x(t), t)) & \leq \ln (V(x(0), 0))+\left(\beta_{2}+1\right) t+\frac{2}{\varepsilon} \ln (n)+\frac{\varepsilon-1}{2} \int_{0}^{t} \frac{\left\|V_{x}(x(s), s) g(x(s), s)\right\|^{2}}{V^{2}(x(s), s)} d s \\
& \leq \ln (V(x(0), 0))+\left(\beta_{2}+1\right) t+\frac{2}{\varepsilon} \ln (n)-\frac{1-\varepsilon}{2} \int_{0}^{t} \frac{\varrho(s) V^{2}(x(s), s)+\zeta}{V^{2}(x(s), s)} d s \\
& \leq \ln (V(x(0), 0))+\left(\beta_{2}+1\right) t+\frac{2}{\varepsilon} \ln (n)-\frac{1-\varepsilon}{2} \int_{0}^{t} \varrho(s) d s-\frac{1-\varepsilon}{2} \int_{0}^{t} \frac{\zeta}{V^{2}(x(s), s)} d s
\end{aligned}
$$

From condition (5.6), there exists $\beta_{3} \in \mathbb{R}_{+}$such that

$$
\lim _{t \longrightarrow \infty} \sup \frac{\int_{0}^{t} \varrho(s) d s}{t} \leq \beta_{3}
$$


then there exists $\sigma>0$ such that

$$
\int_{0}^{t} \varrho(s) d s \leq\left(\beta_{3}+\sigma\right) t
$$

Since $0<\varepsilon<1$, the following inequality

$$
-\frac{1-\varepsilon}{2} \int_{0}^{t} \frac{\zeta}{V^{2}(x(s), s)} d s \leq 0
$$

Then inequality (5.6) becomes

$$
\ln (V(x(t), t)) \leq \ln \left(V(x(0), 0)+\left(\beta_{2}+1\right) t+\frac{2}{\varepsilon} \ln (n)-\frac{1-\varepsilon}{2}\left(\beta_{3}+\sigma\right) t .\right.
$$

Consequently, for almost all $\omega \in \Omega$, if $n-1 \leq t \leq n$ and $n \geq n_{0}$,

$$
\frac{1}{t} \ln (V(x(t), t)) \leq-\frac{1}{2}\left[(1-\varepsilon)\left(\beta_{3}+\sigma\right)-2\left(\beta_{2}+1\right)\right]+\frac{\ln (V(x(0), 0))+\frac{2}{\varepsilon} \ln (n)}{n-1} .
$$

This implies,

$$
\lim _{t \rightarrow+\infty} \sup \frac{1}{t} \ln (V(x(t), t)) \leq-\frac{1}{2}\left[(1-\varepsilon)\left(\beta_{3}+\sigma\right)-2\left(\beta_{2}+1\right)\right] \quad \text { a.s. }
$$

Putting $\varepsilon \longrightarrow 0$ gives,

$$
\lim _{t \rightarrow+\infty} \sup \frac{1}{t} \ln \left(\left\|y\left(t ; t_{0}, x_{0}\right)\right\|-\left(\frac{\gamma}{\beta_{1}}\right)^{\frac{1}{p}}\right) \leq \frac{\left(-\left(\beta_{3}+\sigma\right)-2\left(\beta_{2}+1\right)\right)}{2} \quad \text { a.s. }
$$

If the inequality $\left(\beta_{3}+\sigma\right)>2\left(\beta_{2}+1\right)$ is satisfied, then the system (2.1) is almost surely globally practically uniformly exponentially stable with respect to $y$.

Now, we provide an illustrative example that implement the previous result.

Example 5.4. In this example, the second equation is exponentially stable, so, I guess that my comments in red in the first example are also valid here, I mean, I think that this example is stable in all the variables. Please, we need to find an example in which we cannot prove stability in all the variables, but only in a few of them.

Consider the following stochastic system:

$$
\left\{\begin{array}{l}
d x_{1}(t)=f(x(t)) d t+G x_{1}(t) d B_{t} \\
d x_{2}(t)=\alpha x_{2}(t) d t+\beta x_{2}(t) d B_{t}, \quad \alpha+\frac{\beta^{2}}{2}<0
\end{array}\right.
$$


where $x=\left(x_{1}, x_{2}\right) \in \mathbb{R}^{3}$, and $x_{1}=\left(z_{1}, z_{2}\right) \in \mathbb{R}^{2}, B(t)$ is one-dimensional Brownian motion,

$$
f(x)=\left(\begin{array}{c}
\left(z_{1}+1\right) \cos \left(z_{2}\right) \\
\left(z_{2}+1\right) \sin \left(x_{2}\right),
\end{array}\right), \quad G=\left(\begin{array}{cc}
4 & -0.4 \\
-0.4 & r
\end{array}\right) .
$$

With initial value $x_{0}=\left(x_{10}, x_{20}\right)$, and $x_{10}=\left(z_{10}, z_{20}\right)$.

It is clear that, for $\alpha+\frac{\beta^{2}}{2}<0, x_{2}\left(t, t_{0}, x_{0}\right)$ is stochastically bounded since $x_{2}\left(t, t_{0}, x_{0}\right)$ is geometric Brownian motion (see [16]).

Consider the Lyapunov-like function: $V(t, x)=x_{1}^{2}=z_{1}^{2}+z_{2}^{2}$.

It is easy to verify that

$$
\begin{aligned}
L V(x, t) & =2 z_{1}\left(z_{1}+1\right) \cos \left(z_{2}\right)+2 z_{2}\left(z_{2}+1\right) \sin \left(x_{2}\right)+\left\|G x_{1}\right\|^{2} \\
& \leq 2 z_{1}\left(z_{1}+1\right)+2 z_{2}\left(z_{2}+1\right)+\left\|G x_{1}\right\|^{2} \\
& \leq 22.36 x_{1}^{2}+2
\end{aligned}
$$

and

$$
\left\|V_{x} G x_{1}\right\|^{2}=\left\|2 x_{1}^{T} G x_{1}\right\|^{2} \geq 51.84\left\|x_{1}\right\|^{4}+0 .
$$

Thus, the constants in Theorem 5.1 become $\beta_{1}=1, \beta_{2}=22.36, \beta_{3}=51.84, p=\gamma=2$, $\zeta=\sigma=0$. Clearly, $\beta_{3}>2\left(\beta_{2}+1\right)$.

Note that we cannot apply the result of [10] to deduce the practical uniform exponential stability of the system, because $V$ is not definite positive, Mohamed, indeed we cannot apply the results in [10] to this precise Lyapunov function $V$ but it is possible that we can apply to another $V$, so it is not fully justified that our problem does not have stability in all the variables. Please, we need an example in which we can ensure that stability in all variables does not hold. it is only definite positive with respect to $x_{1}$. Therefore, all conditions of Theorem (5.1) are satisfied, hence the system (5.9) is almost surely globally practically uniformly exponentially stable with respect to $x_{1}$ with $r=\sqrt{2}$.

\section{References}

[1] M. Anabtawi, Practical Stability of Nonlinear Stochastic Hybrid Parabolic Systems of ItoType: Vector Lyapunov Functions Approach, Nonlinear Analysis: Real World Applications, Volume 12, Issue 3, (2011) 1386-1400.

[2] L. Arnold, Stochastic differential equations: Theory and Applications, John Wiley, New York-London-Sydney, 1974.

[3] E. Awad, F.E.C. Culick, On the existence and stability of limit cycles for longitunical acoustic modes in a combustion chamber, Comb. Scien. Tech., Izd. MAI: vol. 46,(1986), 195-222.

[4] B. Ben Hamed, I. Ellouze, M.A. Hammami, Practical uniform stability of nonlinear differential delay equations. Mediterranean Journal of Mathematics, 8 (2011), no. 4, 603-616. 
[5] A. BenAbdallah, M. Dlala, M. A. Hammami, A new Lyapunov function for perturbed non linear systems, Systems and Control letters, 56 (3) (2007) 179-187.

[6] A. BenAbdallah, I. Ellouze and M. A. Hammami, Practical stability of nonlinear time-varying cascade systems, Journal of Dynamical and Control Systems, Vol. 15, No. 1, (2009) 4562.

[7] A. Ben Makhlouf, Stability with respect to part of the variables of nonlinear Caputo fractional differential equations, Mathematical Communications, Math. Commun. 23(2018), 119-126

[8] T. Caraballo, On the decay rate of solutions of non-autonomous differential systems, Electron. J. Diff. Eqns. 2001, 2001 (05), 1-17.

[9] T. Caraballo, María J. Garrido-Atienza, José Real, Stochastic stabilization of differential systems with general decate rate, Systems and Control Letters, 48 (2003), 397-406.

[10] T. Caraballo, M. A. Hammami, L. Mchiri, On the practical global uniform asymptotic stability of stochastic differential equations, Stochastics an international journal of probability and stochastic processes, vol. 88, 2016, 45-56.

[11] S. S. Dragomir, Some Gronwall type inequalities and applications, (2003).

[12] W.M. Haddad, V. Chellaboina, Nonlinear Dynamical Systems and Control, (2007).

[13] R. Z.Has'minskii, Stochastic Stability of Differential Equations, Sijthoff Noordhoff, (1980).

[14] H. K. Khalil, Nonlinear Systems, Mac-Millan, 2nd edition, (1996).

[15] X. Mao, Exponential Stability of Stochastic Differential Equations, Marcel Dekker, Inc.: New York, (1994).

[16] X. Mao, Stochastic Differential Equations and Applications, Ellis Horwood, Chichester, U.K, (1997).

[17] B. Oksendal, Stochastic Differential Equations: an Introduction with Applications (6th ed.) Springer-Verlag, New York, 2003.

[18] U. H. Thygesen, A survey of Lyapunov Techniques for Stochastic Differential Equations, IMM Technical Report n.c, (1997).

[19] O.Ignatyev, Partial asymptotic stability in probability of stochastic differential equations, Statistics and Probability Letters, 79 (2009), 597-601.

[20] K.-Y. Lum, D.S. Bernstein, V.T. Coppola, Global stabilization of the spinning top with mass imbalance, Dynamics and Stability of Systems, 10 vol. 10, (1995), 339-365.

[21] N. Rouche, P. Habets, M. Lalog, Stability Theory by Liapunov's Direct Method. New York: Springer, 1977. 
[22] V.A. Sinitsyn, On stability of solution in inertial navigation problem, Certain Problems on Dynamics of Mechanical Systems (in Russian), Izd. MAI: Moscow, (1991), 46-50.

[23] V.I. Zubov, The Dynamics of Controlled Systems. Moscow: Vysshaya Shkola, (1982).

[24] V. I. Vorotnikov, Partial Stability and Control, (1998). 